\title{
Anhang - Verlaufspläne für die Lehr-Lern-Bausteine
}

Brodesser, Ellen [Hrsg.]; Frohn, Julia [Hrsg.]; Welskop, Nena [Hrsg.]; Liebsch, Ann-Catherine [Hrsg.]; Moser, Vera [Hrsg.]; Pech, Detlef [Hrsg.]: Inklusionsorientierte Lehr-Lern-Bausteine für die Hochschullehre. Ein Konzept zur Professionalisierung zukünttiger Lehrkräfte. Bad Heilbrunn : Verlag Julius Klinkhardt 2020, S. 193-200. (Interdisziplinäre Beiträge zur Inklusionsforschung)

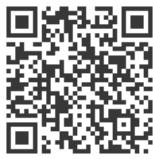

Quellenangabe/ Reference:

Anhang - Verlaufspläne für die Lehr-Lern-Bausteine - In: Brodesser, Ellen [Hrsg.]; Frohn, Julia [Hrsg.]; Welskop, Nena [Hrsg.]; Liebsch, Ann-Catherine [Hrsg.]; Moser, Vera [Hrsg.]; Pech, Detlef [Hrsg.]: Inklusionsorientierte Lehr-Lern-Bausteine für die Hochschullehre. Ein Konzept zur Professionalisierung zukünftiger Lehrkräfte. Bad Heilbrunn : Verlag Julius Klinkhardt 2020, S. 193-200 - URN:

urn:nbn:de:0111-pedocs-190291 - DOI: 10.25656/01:19029

https://nbn-resolving.org/urn:nbn:de:0111-pedocs-190291

https://doi.org/10.25656/01:19029

in Kooperation mit / in cooperation with:

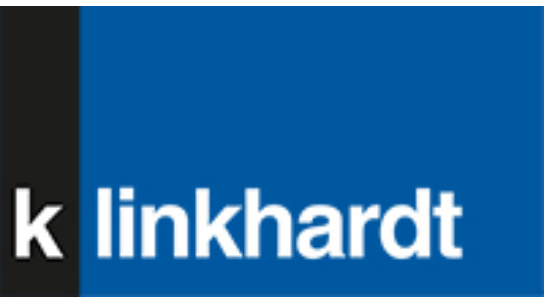

http://www.klinkhardt.de

\section{Nutzungsbedingungen}

Dieses Dokument steht unter folgender Creative Commons-Lizenz: http://creativecommons.org/licenses/by-nc-sa/4.0/deed.de - Sie dürfen das Werk bzw. den Inhalt unter folgenden Bedingungen vervielfältigen, verbreiten und öffentlich zugänglich machen sowie Abwandlungen und Bearbeitungen des Werkes bzw. Inhaltes anfertigen: Sie müssen den Namen des Autors/Rechteinhabers in der von ihm festgelegten Weise nennen. Dieses Werk bzw. der Inhalt darf nicht für kommerzielle Zwecke verwendet werden. Die neu entstandenen Werke bzw. Inhalte dürfen nur unter Verwendung von Lizenzbedingungen weitergegeben werden, die mit denen dieses Lizenzbedingungen weitergegeben werden,
Lizenzvertrages identisch oder vergleichbar sind.

Mit der Verwendung dieses Dokuments erkennen Sie die Nutzungsbedingungen an.

\section{Terms of use}

This document is published under following Creative Commons-License: http://creativecommons.org/licenses/by-nc-sa/4.0/deed.en - You may copy, distribute and transmit, adapt or exhibit the work in the public and alter, transform or change this work as long as you attribute the work in the manner specified by the author or licensor. You are not allowed to make commercial may distribute the resulting work only under this or a comparable license.

By using this particular document, you accept the above-stated conditions of use.

\section{Kontakt / Contact:}

\section{peDOCS}

DIPF | Leibniz-Institut für Bildungsforschung und Bildungsinformation Informationszentrum (IZ) Bildung

E-Mail: pedocs@dipf.de

Internet: www.pedocs.de

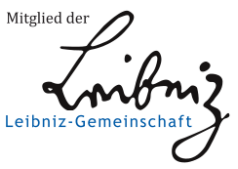


Interdisziplinäre Beiträge zur Inklusionsforschung

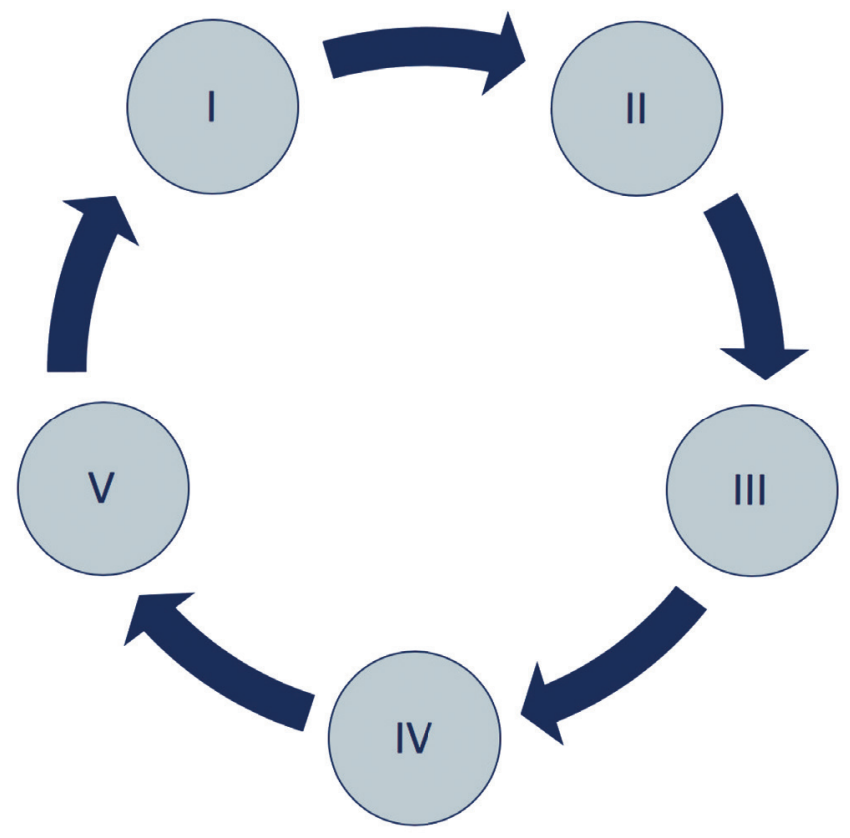

Ellen Brodesser / Julia Frohn /

Nena Welskop / Ann-Catherine Liebsch /

Vera Moser / Detlef Pech

(Hrsg.)

\section{Inklusionsorientierte Lehr-Lern- Bausteine für die Hochschullehre}

Ein Konzept zur Professionalisierung zukünftiger Lehrkräfte 
Brodesser / Frohn / Welskop / Liebsch / Moser / Pech Inklusionsorientierte Lehr-Lern-Bausteine für die Hochschullehre 
Interdisziplinäre Beiträge zur Inklusionsforschung

herausgegeben von Marina Egger, Jullia Frohn,

Vera Moser und Detlef Pech 


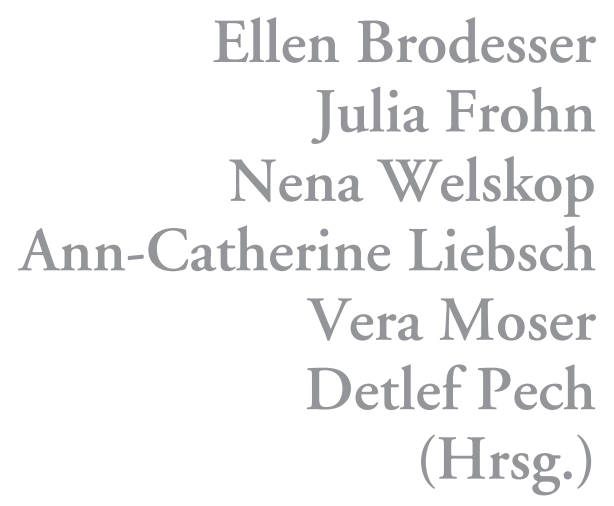

\section{Inklusionsorientierte Lehr-Lern- Bausteine für die Hochschullehre}

Ein Konzept zur Professionalisierung zukünftiger Lehrkräfte 
Das diesem Buch zugrundeliegende Vorhaben wurde im Rahmen der gemeinsamen „Qualitätsoffensive Lehrerbildung“ von Bund und Ländern mit Mitteln des Bundesministeriums für Bildung und Forschung unter dem Förderkennzeichen 01JA1620 gefördert.

Die Verantwortung für den Inhalt der Veröffentlichung liegt bei den Autor*innen.

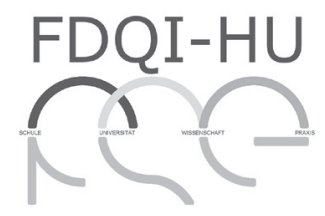

GEFÖRDERT VOM

Bundesministerium
für Bildung
und Forschung

Dieser Titel wurde in das Programm des Verlages mittels eines Peer-Review-Verfahrens aufgenommen.

Für weitere Informationen siehe www.klinkhardt.de.

Bibliografische Information der Deutschen Nationalbibliothek

Die Deutsche Nationalbibliothek verzeichnet diese Publikation in der Deutschen Nationalbibliografie; detaillierte bibliografische Daten

sind im Internet abrufbar über http://dnb.d-nb.de.

2020.i. (c) by Julius Klinkhardt.

Satz und Grafik Umschlagseite 1: Kay Fretwurst, Spreeau.

Druck und Bindung: AZ Druck und Datentechnik, Kempten.

Printed in Germany 2020.

Gedruckt auf chlorfrei gebleichtem alterungsbeständigem Papier.

(c)(i)(5) Die Publikation (mit Ausnahme aller Fotos, Grafiken und Abbildungen) ist veröffentlicht unter der Creative Commons-Lizenz: CC BY-NC-SA 4.0 International

https://creativecommons.org/licenses/by-nc-sa/4.0/

ISBN 978-3-7815-5798-7 Digital

doi.org/10.35468/5798

ISBN 978-3-7815-2361-6 Print 


\section{Inhalt}

1 Einführung: Inklusionsorientierte Lehr-Lern-Bausteine für die Hochschullehre eine Begründung durch Praxisbezug, Theorie und Methodik ............... Ellen Brodesser, Julia Frohn, Nena Welskop, Ann-Catherine Liebsch, Vera Moser und Detlef Pech

2 Heterogenitätssensibilität, adaptive Lehrkompetenz und Sprachbildung als Ausgangspunkte für die Entwicklung

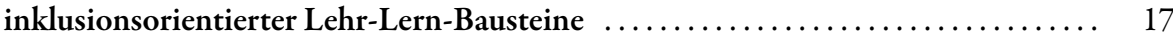

2.1 Heterogenitätssensibilität als Voraussetzung adaptiver Lehrkompetenz ....... 19 Nena Welskop und Vera Moser

2.2 Lehrkräfteprofessionalisierung: adaptive Lehrkompetenz für inklusiven Unterricht Julia Frohn, Lena Schmitz und Hans Anand Pant

2.3 Anschlussstellen zwischen Sprachbildung und adaptiver Lehrkompetenz für den inklusiven Unterrich Julia Frohn und Laura Rödel

3 Inklusionsorientierte Lehr-Lern-Bausteine: theoretische Einbettung, didaktische Kommentierung und Einsatz in der inklusionssensiblen Hochschullehre

3.1 Einleitung: Entwicklung und Umsetzung inklusionsorientierter Lehr-Lern-Bausteine Ann-Catherine Liebsch

3.2 Der Baustein Heterogenitätssensibilität: inklusionspädagogische Grundlegung

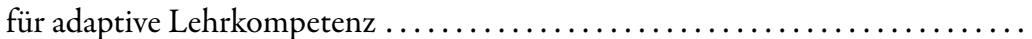
Dietlind Gloystein

3.3 Der Baustein Adaptive diagnostische Kompetenz: ein Selbstversuch und inklusionssensible pädagogische Diagnostik als Impuls für Perspektivwechsel und professionelle Reflexion

Dietlind Gloystein und Julia Frohn

3.4 Der Baustein Adaptive didaktische Kompetenz: inklusive (Fach-)Didaktik und adaptive didaktische Kompetenz .......... 76 Fabian Eckert und Ann-Catherine Liebsch

3.5 Der Baustein Adaptive Klassenführungskompetenz: effektive Klassenführung als Basis für den inklusiven Unterricht ........... Ann-Catherine Liebsch und Yasmin Patzer

3.6 Der Baustein Sprachbildung: ein Lehr-Lern-Angebot für die inklusionssensible fachdidaktische Lehre Laura Rödel 
4 Multiperspektivische Evaluation $\ldots \ldots \ldots \ldots \ldots \ldots \ldots \ldots \ldots \ldots \ldots \ldots \ldots, 111$

4.1 Heterogenitätssensibilität angehender Lehrkräfte: empirische Ergebnisse . .... 113 Lena Schmitz, Toni Simon und Hans Anand Pant

4.2 Adaptive Lehrkompetenz: Bildung von Indizes und empirische Ergebnisse zur Wirkung universitärer Lehrveranstaltungen ...... 124 Lena Schmitz, Ellen Brodesser und Hans Anand Pant

4.3 Den üblichen Weg verlassen. Objektiv-hermeneutische Analyse der Interviews mit Dozierenden zum Einsatz inklusionsorientierter Lehr-Lern-Bausteine in fachdidaktischen Seminaren ................. 13 Ellen Brodesser, Nena Welskop und Julia Frohn

5 Ausblick: Inklusionsorientierung in verschiedenen Phasen der Lehrkräftebildung

5.1 Potenziale inklusionsorientierter Lehr-Lern-Bausteine für die Übertragung auf verschiedene Fachdidaktiken und für die MINT-Fächer . ........... 151 Yasmin Patzer, Julia Frohn und Niels Pinkwart

5.2 Aufbau und Erweiterung von Heterogenitätssensibilität und diagnostischer Kompetenz durch inklusionsorientierte Lehr-Lern-Bausteine in der universitären Lehrkräftebildung: Einordnung und Weiterentwicklung der konzipierten Unterrichtseinheiten aus inklusionspädagogischer Sicht ........ 162 Dietlind Gloystein und Vera Moser

5.3 Impulse aus der Konzeption der inklusionsorientierten Lehr-Lern-Bausteine und aus dem Forschenden Lernen für die Sprachbildung in der ersten Phase der Lehrkräftebildung . . Laura Rödel, Maria Große und Constanze Saunders

5.4 Einsatzpotenziale inklusionsorientierter Lehr-Lern-Bausteine im Vorbereitungsdienst und in der Berliner Mentoringqualifizierung ............ Nena Welskop, Ellen Brodesser und Caroline Körbs

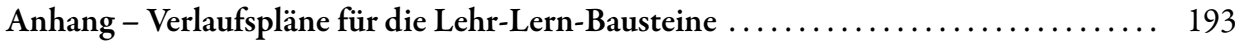

1. Verlaufsplan für den Baustein Heterogenitätssensibilität ................. 194

2. Verlaufsplan für den Baustein Adaptive diagnostische Kompetenz . . . . . . . . . . 195

3. Verlaufsplan für den Baustein Adaptive didaktische Kompetenz . . . . . . . . . . . 197

4. Verlaufsplan für den Baustein Adaptive Klassenführungskompetenz .......... 198

5. Verlaufsplan für den Baustein Sprachbildung ...................... 199

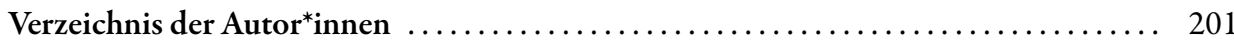




\section{Anhang - Verlaufspläne für die Lehr-Lern-Bausteine}

Im Folgenden werden die Verlaufspläne der fünf inklusionsorientierten Bausteine für die Hochschullehre in den Fachdidaktiken aufgeführt, wie sie in den Beiträgen 3.2 bis 3.6 beschrieben werden. Alle Bausteine sind als flexible, adaptierbare Lehr-Lern-Formate konzipiert, die an die Bedürfnisse der Lehrenden und ihrer Seminargruppen angepasst werden können. Die konkreten inklusionsbezogenen Ziele und die damit verbundenen didaktischen Kommentare sind den Beiträgen 3.2 bis 3.6 zu entnehmen. Dieser Anhang soll einen Überblick über den tatsächlichen Verlauf geben - jedoch nicht isoliert von den entsprechenden Beiträgen verstanden werden.

Materialien zum Einsatz der Bausteine, z.B. Präsentationen, Bilder, finden Sie unter www.huberlin.de/fdqi/Bausteine.

Folgende Abkürzungen werden in den Verlaufsplänen verwendet:

DV Dozent*innenvortrag

EA Einzelarbeit

GA Gruppenarbeit

gSG gelenktes Seminargespräch

TN Teilnehmer*innen 


\section{Verlaufsplan für den Baustein Heterogenitätssensibilität}

(ausführliche didaktische Kommentare siehe Beitrag 3.2 in diesem Band)

\begin{tabular}{|c|c|c|c|}
\hline Phase & Ablauf und Impulse & $\begin{array}{l}\text { Sozial- } \\
\text { form }\end{array}$ & Medien \\
\hline Einstieg & $\begin{array}{l}\text { Der/die Dozent*in zeigt den TN eine Zitrone. } \\
\text { Die TN benennen allgemeine (äußere) Merkmale/Eigenschaften } \\
\text { von Zitronen. } \\
\text { Die Ergebnisse werden visualisiert. }\end{array}$ & gSG & $\begin{array}{l}\text { Zitronen } \\
\text { Tafel }\end{array}$ \\
\hline $\begin{array}{l}\text { Erarbei- } \\
\text { tung }\end{array}$ & $\begin{array}{l}\text { Der/die Dozent*in teilt die Zitronen aus. } \\
\text { Die TN nehmen ihre Zitrone anhand mehrerer Sinne (fühlen, rie- } \\
\text { chen, schmecken) wahr. Anschließend verfassen sie eine Geschichte } \\
\text { (Gedicht, Zeichnung etc.) über diese besondere Zitrone. } \\
\text { Der/die Dozent*in sammelt die Zitronen wieder ein. }\end{array}$ & EA & Zitronen \\
\hline $\begin{array}{l}\text { Siche- } \\
\text { rung }\end{array}$ & $\begin{array}{l}\text { Ausgewählte Ergebnisse werden im Plenum präsentiert. Die TN } \\
\text { suchen ihre Zitrone heraus und nehmen sie wieder an sich. } \\
\text { Die Ergebnisse werden anhand von Leitfragen im Plenum diskutiert. } \\
\text { Impulse: } \\
\text { - (Wie) War es Ihnen möglich, die eigene Zitrone wiederzufinden? } \\
\text { - Was macht Ihre Zitrone einzigartig? } \\
\text { - Welche spezifischen Merkmale sind Ihnen besonders aufgefallen? } \\
\text { - Waren Sie überrascht? } \\
\text { - Warum unterscheidet sich die erste Sammlung von Eigenschaften } \\
\text { von der zweiten Sammlung? }\end{array}$ & gSG & Tafel \\
\hline Transfer & $\begin{array}{l}\text { Im Plenum wird anhand von Leitfragen ein Transfer auf den inklusi- } \\
\text { ven Unterricht angeleitet. } \\
\text { Impulse: } \\
\text { - Welche Thematik wird hier sichtbar? } \\
\text { - Sehen Sie einen Bezug zum Unterricht? } \\
\text { - Welche Kategorisierungen kennen Sie im schulischen Bereich? } \\
\text { - Welche Wirkungen zeigen Kategorisierungen auf Ihr pädagogi- } \\
\text { sches Handeln? }\end{array}$ & gSG & \\
\hline
\end{tabular}




\section{Verlaufsplan für den Baustein Adaptive diagnostische Kompetenz}

(ausführliche didaktische Kommentare siehe Beitrag 3.3 in diesem Band)

\begin{tabular}{|c|c|c|c|}
\hline Phase & Ablauf und Impulse & $\begin{array}{l}\text { Sozial- } \\
\text { form }\end{array}$ & Medien \\
\hline Einstieg & $\begin{array}{l}\text { Der/die Dozent*in kündigt eine (traditionelle) diagnostische Übung an. } \\
\text { Die TN werden willkürlich in zwei Gruppen eingeteilt, die unter- } \\
\text { schiedliche Arbeitsanweisungen erhalten. } \\
\text { Gruppe A: Sie erhalten je drei Farbstifte. Die Rey-Figur wird Ihnen } \\
\text { I min gezeigt. Zeichnen Sie anschließend die Figur aus dem Gedächtnis } \\
\text { nach. Die Farben kennzeichnen die Erinnerung; rot: sichere Erinnerung, } \\
\text { schwarz: unsichere Erinnerung, lila: freie Ergänzungen. } \\
\text { Gruppe B: Beobachten Sie die Handlungen der Zeichner*innen! } \\
\text { Erkennen Sie Motivation, Frustration, Abwehrverhalten, Konzeption, } \\
\text { Ausdauer, Lösungs- und Lernstrategien? }\end{array}$ & gSG & $\begin{array}{l}\text { Präsen- } \\
\text { tation }\end{array}$ \\
\hline $\begin{array}{l}\text { Erarbei- } \\
\text { tung }\end{array}$ & $\begin{array}{l}\text { Der/die Dozent*in leitet die Übung an. Anschließend zeichnet } \\
\text { Gruppe A, Gruppe B beobachtet. } \\
\text { Die Auswertung erfolgt im Plenum anhand von Leitfragen. } \\
\text { Impulse für Gruppe A: } \\
\text { - Was glauben Sie, mit welchem diagnostischen Ziel wurde diese } \\
\text { Übung durchgeführt? } \\
\text { - Wie haben Sie sich in der Situation gefühlt? } \\
\text { - Haben Sie sich beobachtet gefühlt? Wenn ja, wie haben Sie darauf } \\
\text { reagiert? } \\
\text { Impulse für Gruppe B: } \\
\text { - Hinsichtlich welcher Kriterien haben Sie die Teilnehmer*innen } \\
\text { beobachtet? } \\
\text { - Was konnten Sie hinsichtlich der Kriterien beobachten? Was darüber } \\
\text { hinaus? } \\
\text { - Haben Sie sich hinreichend auf die Beobachtungsaufgabe vorbereitet } \\
\text { gefühlt? }\end{array}$ & $\begin{array}{l}\text { EA/ } \\
\text { gSG }\end{array}$ & $\begin{array}{l}\text { Präsen- } \\
\text { tation }\end{array}$ \\
\hline Transfer I & $\begin{array}{l}\text { Im Plenum werden weitere Aspekte anhand von Leitfragen diskutiert. } \\
\text { Impulse: } \\
\text { - Wie transparent darf/soll diagnostisches Handeln für Schüler*innen } \\
\text { sein? } \\
\text { - Welche Werte bestimmen diagnostisches Handeln? } \\
\text { - Wie stark ist pädagogisches Handeln durch eigene schulische Erfah- } \\
\text { rungen geprägt? } \\
\text { - Welche Rolle spielen in diagnostischen Situationen Begriffe wie } \\
\text { Macht und Hierarchie? } \\
\text { - War das Vorgehen „inklusiv“? }\end{array}$ & gSG & $\begin{array}{l}\text { Präsen- } \\
\text { tation }\end{array}$ \\
\hline $\begin{array}{l}\text { Transfer } \\
\text { II }\end{array}$ & $\begin{array}{l}\text { In Gruppen stellen die TN auf der Grundlage der reflektierten } \\
\text { Selbsterfahrung und ihres Vorwissens Überlegungen hinsichtlich der } \\
\text { Qualitätsmerkmale und Ausgestaltung einer an Inklusion orientierten } \\
\text { Diagnostik an. }\end{array}$ & GA & \\
\hline $\begin{array}{l}\text { Siche- } \\
\text { rung }\end{array}$ & $\begin{array}{l}\text { Die Ergebnisse werden im Plenum zusammengetragen und im Hin- } \\
\text { blick auf die weiteren Ausbildungsanforderungen innerhalb des Lehr- } \\
\text { amtsstudiums und für das spätere professionelle Handeln diskutiert. }\end{array}$ & gSG & \\
\hline
\end{tabular}




\begin{tabular}{lll}
\hline Phase & Ablauf und Impulse & $\begin{array}{c}\text { Sozial- Medien } \\
\text { form }\end{array}$ \\
\hline & $\begin{array}{l}\text { Ausgehend von Leitfragen werden im Plenum Zusammenhänge zwi- } \\
\text { schen einer allgemeinen inklusionssensiblen und einer fachbezogenen } \\
\text { Diagnostik hergestellt. }\end{array}$ & \\
& Impulse: \\
optional: & - Wie können die Schüler*innen mit ihren sehr unterschiedlichen & \\
Transfer & Voraussetzungen am inklusiven Fachunterricht partizipieren? \\
& - Wie können gemeinsame Lernsituationen, ausgehend von der \\
& Grundannahme heterogener Lerngruppen, gestaltet werden? \\
& - Woran kann ich mich bei der Gestaltung meines inklusiven Fachun- \\
& terrichts orientieren, wenn ich diagnostische Informationen nutze?
\end{tabular}




\section{Verlaufsplan für den Baustein Adaptive didaktische Kompetenz}

(ausführliche didaktische Kommentare siehe Beitrag 3.4 in diesem Band)

\begin{tabular}{|c|c|c|c|}
\hline Phase & Ablauf und Impulse & Sozialform & Medien \\
\hline Einstieg & $\begin{array}{l}\text { Metaplanmethode (die TN beantworten stichwortartig } \\
\text { Fragen auf Moderationskarten). } \\
\text { Impuls: Was leistest (Fach-)Didaktik? } \\
\text { Die Antworten werden gesammelt. } \\
\text { Impuls: Wo liegen die Herausforderungen des Unterrich- } \\
\text { tens in heterogenen Lerngruppen? } \\
\text { Die Antworten werden gesammelt. } \\
\text { Die Karten werden im Plenum kommentiert und } \\
\text { thematisch sortiert. }\end{array}$ & $\begin{array}{l}\text { EA/ } \\
\text { gSG }\end{array}$ & $\begin{array}{l}\text { Moderations- } \\
\text { karten } \\
\text { Tafel }\end{array}$ \\
\hline Darbietung & $\begin{array}{l}\text { Der/die Dozent*in hält einen präsentationsgestützten } \\
\text { Kurzvortrag. } \\
\text { Themen: inklusive Didaktik, DiMiLL, adaptive didakti- } \\
\text { sche Kompetenz }\end{array}$ & DV & Präsentation \\
\hline Erarbeitung & $\begin{array}{l}\text { Die TN entwickeln und diskutieren in Gruppen anhand } \\
\text { von didaktischen Dreiecken aus Prozessmerkmalen, } \\
\text { Strukturelementen und fachdidaktischen Bedingungen } \\
\text { des DiMiLL Fragen, die sich in diesem Zusammenhang } \\
\text { ergeben. }\end{array}$ & GA & \\
\hline Sicherung & $\begin{array}{l}\text { Im Plenum werden der Metaplan und die aufgeworfenen } \\
\text { Fragen zueinander in Beziehung gesetzt. } \\
\text { Abschließend werden die Komponenten der didaktischen } \\
\text { Kompetenz zusammengestellt. }\end{array}$ & gSG & $\begin{array}{l}\text { Metaplan } \\
\text { (s.o.) }\end{array}$ \\
\hline
\end{tabular}




\section{Verlaufsplan für den Baustein Adaptive Klassenführungskompetenz}

(ausführliche didaktische Kommentare siehe Beitrag 3.5 in diesem Band)

\begin{tabular}{|c|c|c|c|}
\hline Phase & Ablauf und Impulse & Sozialform & Medien \\
\hline Einstieg & $\begin{array}{l}\text { (Mehrmaliges) Zeigen eines Filmausschnitts aus } \\
\text { „Die Klasse“. } \\
\text { Impulse: } \\
\text { - Was haben Sie gesehen? Beschreiben Sie die } \\
\text { Situation. } \\
\text { - Wie schätzen Sie die Situation ein? Warum verhält } \\
\text { sich Monsieur Marin so? Welche Strategien setzt } \\
\text { er ein? } \\
\text { - Was halten Sie vom Verhalten des Lehrers? } \\
\text { - Wie erfolgreich ist Monsieur Marin mit seinen } \\
\text { Strategien und Impulsen? } \\
\text { - Was hat Ihnen gefallen, was nicht? }\end{array}$ & gSG & $\begin{array}{l}\text { Film/ } \\
\text { Präsentation }\end{array}$ \\
\hline Erarbeitung I & $\begin{array}{l}\text { Die TN entwickeln Handlungsalternativen zu dem } \\
\text { im Film gezeigten Lehrerhandeln. } \\
\text { Impulse: } \\
\text { - An welchen Stellen hätte sich Monsieur Marin } \\
\text { anders verhalten können? } \\
\text { - Wie hätten Sie sich verhalten? } \\
\text { - Welche Alternativen gibt es zum Vorgehen des } \\
\text { Lehrers? }\end{array}$ & gSG & Präsentation \\
\hline Darbietung & $\begin{array}{l}\text { Der/die Dozent*in hält einen präsentationsgestütz- } \\
\text { ten Kurzvortrag. } \\
\text { Themen: Definition „Klassenführung“, Techniken } \\
\text { und Strategien, Anbindung an DiMiLL }\end{array}$ & DV & Präsentation \\
\hline $\begin{array}{l}\text { optional: } \\
\text { Erarbeitung II }\end{array}$ & $\begin{array}{l}\text { Die TN erarbeiten arbeitsteilig Strategien zur Klas- } \\
\text { senführung, die für den jeweiligen Fachunterricht } \\
\text { besondere Relevanz besitzen. }\end{array}$ & GA & \\
\hline $\begin{array}{l}\text { optional: } \\
\text { Sicherung }\end{array}$ & $\begin{array}{l}\text { Ggf. werden die Ergebnisse im Plenum zusammenge- } \\
\text { tragen und strukturiert. } \\
\text { Abschließend werden der Zusammenhang von Klas- } \\
\text { senführung und (Fach-) Unterricht in heterogenen } \\
\text { Lerngruppen im Plenum diskutiert. }\end{array}$ & gSG & \\
\hline
\end{tabular}




\section{Verlaufsplan für den Baustein Sprachbildung}

(ausführliche didaktische Kommentare siehe Beitrag 3.6 in diesem Band)

\begin{tabular}{|c|c|c|c|}
\hline Phase & Ablauf und Impulse & Sozialform & Medien \\
\hline Einstieg & $\begin{array}{l}\text { Sensibilisierungsübung: Die TN bearbeiten nach } \\
\text { dem Prinzip Seitenwechsel eine Aufgabe in ihrer } \\
\text { zweitbesten Sprache. } \\
\text { Impulse: } \\
\text { - Schätzen Sie: Wie viel Prozent Ihrer Aufmerksam- } \\
\text { keit haben Sie bei der Bearbeitung der Aufgabe auf } \\
\text { das fachliche Problem und wie viel Prozent auf die } \\
\text { Sprache gelegt? } \\
\text { - Hatten Sie Probleme bei der Bearbeitung der } \\
\text { Aufgabe? Wenn ja, welche? } \\
\text { - Welche Hilfsmittel hätten Ihnen ggf. geholfen? } \\
\text { - Wie haben Sie sich in dieser Situation gefühlt? }\end{array}$ & $\begin{array}{l}\text { EA/ } \\
\text { gSG }\end{array}$ & $\begin{array}{l}\text { auf ein } \\
\text { Fachthema } \\
\text { bezogener } \\
\text { Impuls/ } \\
\text { Präsentation }\end{array}$ \\
\hline Darbietung & $\begin{array}{l}\text { Der/die Dozent*in hält einen präsentationsgestütz- } \\
\text { ten Kurzvortrag. } \\
\text { Themen: Sprachbildung, Bildungssprache, Scaffol- } \\
\quad \text { ding } \\
\text { Der/die Dozent*in bezieht die TN durch Reflexions- } \\
\text { fragen mit ein. } \\
\text { Impulse: } \\
\text { - Was verstehen Sie unter Sprachbildung? } \\
\text { - Was bedeutet, Fachunterricht sprachsensibel zu } \\
\text { gestalten? } \\
\text { - Wie hängen die Konzepte „Bildungssprache“ und } \\
\text { „Scaffolding“ zusammen? } \\
\text { - Welche Asssoziationen begleiten die Metapher des } \\
\text { Baugerüstes? } \\
\text { - Welche eigenen Erfahrungen konnten Sie bereits } \\
\text { mit Scaffolding sammeln und wie bewerten Sie } \\
\text { diese? }\end{array}$ & $\begin{array}{l}\mathrm{DV} / \\
\mathrm{gSG}\end{array}$ & Präsentation \\
\hline Erarbeitung & $\begin{array}{l}\text { Die TN bearbeiten in Gruppen fachspezifisches } \\
\text { sprachbildendes Material anhand von Leitfragen. } \\
\text { Impulse: } \\
\text { - Welche Elemente des Scaffoldings erkennen Sie } \\
\text { wieder? } \\
\text { - Wie wird im sprachbildenden Material fachliches } \\
\text { und sprachliches Lernen miteinander verknüpft? } \\
\text { - Welche sprachlichen Prozesse werden unterstützt/ } \\
\text { angeregt? } \\
\text { - Welche Vorkenntnisse benötigen Lernende fach- } \\
\text { lich und sprachlich, um mit dem Material arbeiten } \\
\text { zu können? } \\
\text { - Was wäre ggf. für einen inklusionssensiblen Unter- } \\
\text { richt noch wünschenswert? }\end{array}$ & GA & \\
\hline
\end{tabular}




\begin{tabular}{llcc}
\hline Phase & Ablauf und Impulse & Sozialform & Medien \\
\hline \multirow{2}{*}{ Sicherung } & $\begin{array}{l}\text { Das Material wird anhand der o.g. Leitfragen im } \\
\text { Plenum ausgewertet. }\end{array}$ & gSG & \\
\hline & Abschließend werden der Zusammenhang von & \\
& Sprachbildung und Inklusion im Plenum diskutiert. & \\
& Impulse: \\
& - Wie hängen Inklusion und Sprachbildung zusam- & \\
& men? & \\
& - Auf welche Weise kann die sprachsensible Gestal- & gSG \\
& tung des Fachunterrichts zur Inklusion beitragen? & \\
- Welche blinden Flecken zeigen sich in der Sprach- & \\
& bildung in Hinblick auf inklusiven Fachunterricht? & \\
& - Welche Prozessmerkmale und Strukturelemente & \\
& des DiMiLL berührt die Sprachbildung? & \\
& &
\end{tabular}

